\title{
Full polarization optical profilometry
}

\author{
S. Arhab, ${ }^{*}$ H. Giovannini, K. Belkebir, and G. Soriano \\ Institut Fresnel (CNRS UMR 7249), Aix-Marseille Université, Campus de St Jérôme, 13013 Marseille, France \\ ${ }^{*}$ Corresponding author: slimane.arhab@fresnel.fr
}

Received February 24, 2012; revised May 23, 2012; accepted May 23, 2012; posted May 25, 2012 (Doc. ID 163610); published July 11, 2012

\begin{abstract}
Optical digital tomographic microscopy can be used for profilometry. The profile of the surface can be estimated from measurements of the complex diffracted far field obtained when the sample is illuminated successively under various incidences. Outside the validity domain of perturbative theories of diffraction, the profile is determined by using an iterative inverse wave scattering numerical method. In this paper we show that, for perfectly conducting surfaces, the two fundamental polarization cases involve different distances of interaction in the multiple scattering phenomenon. The use of both polarization cases in the inversion process leads to a considerable improvement of the lateral resolution. Robustness to noise is also discussed. (c) 2012 Optical Society of America OCIS codes: $\quad 290.3200,050.1755,290.4210,290.5880,120.6660$.
\end{abstract}

\section{INTRODUCTION}

Optical digital tomographic microscopy (ODTM) is a quantitative three-dimensional imaging technique [1] in which the optogeometrical parameters of the object (permittivity, shape) are determined numerically by solving iteratively an inverse scattering problem. The data used in the numerical reconstruction of the object are the measurements of the field diffracted by the object illuminated successively under different angles [2]. In the inversion algorithm, different diffraction theories can be considered for describing the interaction between the electromagnetic field and the object. When the optogeometrical parameters of the object such as the Born approximation are valid, the object can be reconstructed directly by calculating a Fourier transform (FT) of the diffracted field. Different papers [3] $4-6$ ] have reported theoretical and experimental results showing that, in this case, the synthetic aperture obtained by varying the incidence angle can lead to a resolution twice better than that of conventional microscopes. Outside the domain of validity of first-order approximations, when multiple scattering occurs in the sample, a resolution beyond the Abbe-Rayleigh criterion can be reached [7-11]. In this case, an inversion procedure based on a rigorous model of diffraction is mandatory. Performances of ODTM in terms of resolution and its capability to reconstruct objects with strong slopes and high permittivity contrasts show that it is potentially an interesting alternative to classical optical profilometry based on Mirau, Linnik, or Michelson interferometers.

A preliminary study of the applicability of ODTM to profilometry in the resonance domain has been presented in a previous paper [12]. The importance of using a rigorous method for calculating the scattered field has been stressed, and it has been shown that ODTM can be applied when high lateral resolutions are required. The discussion was made only for surfaces illuminated under TE polarization (electric component perpendicular to the plane of incidence). In this paper we extend the study to both polarization cases. We have developed an inversion algorithm for the TM polarization (magnetic component perpendicular to the plane of incidence) in the perfectly conducting case. We show that better reconstructions with increased resolution can be obtained when the data measured for both polarizations are processed by inversion algorithms accounting for TE and TM cases. We show in particular that the best results are obtained when the profile reconstructed in TE polarization is used as a starting estimate for the inversion algorithm in TM polarization. We also give a physical interpretation of the superresolved reconstruction reached in the multiple scattering regime. In particular, we analyze the role of the distance of interaction between the scatterers on the surface in the far-field measurement.

In Section (2) we describe the scattering problem and we present the geometry considered. Section (3) is devoted to the description of the boundary integral equation method used for calculating rigorously the field scattered by rough surfaces in TE and TM polarizations. The numerical iterative algorithm used for reconstructing the surface profile from the scattered field is described in Section (4). In Section (5) we present the results of numerical simulations. We analyze the influence of the distance of interaction between the scatterers on the subwavelength resolution obtained in the multiple scattering regime. We present some numerical reconstructions of randomly rough surfaces. Robustness against the presence of noise in the data as well as against errors of positioning angles of detection are also reported.

\section{DIRECT SURFACE WAVE SCATTERING PROBLEM}

For the scattering of an electromagnetic wave from a rough surface at a given pulsation $\omega$, when the surface height and the incident field share an invariance direction, two fundamental polarization cases appear, which correspond to two independent scalar two-dimensional scattering problems. These problems are denoted TE and TM, depending on whether the electric field or the magnetic field is set parallel to the invariance direction. In this paper, this direction fits the $y$ axis of a Cartesian coordinate system $(x, y, z)$ of origin $O$ and thus $(x O z)$ in the plane of incidence.

Assume that a surface $\Gamma: z=\eta(x)$ characterized by its variation of the height $\eta: x \rightarrow \eta(x)$ is successively illuminated 
from air, the incident medium, by $L$ different beams centered on angles $\theta_{l}$ and for each illumination $l$ the scattered far field is measured for $M$ different angles of detection $\theta_{m}$. All beams share the same width, defined by the so-called tapering parameter $g$ [13]. In the far field, with a time dependency $e^{-i \omega t}$ and wavenumber $k=\frac{\omega}{c}=\frac{2 \pi}{\lambda}$, at point $\mathbf{r}_{m}=\left(x_{m}, z_{m}\right)=$ $\left(\left|\mathbf{r}_{m}\right| \sin \theta_{m},\left|\mathbf{r}_{m}\right| \cos \theta_{m}\right)$ in vacuum along the direction $\theta_{m}$, the scattered field writes as a cylindrical wave:

$$
\psi^{\mathrm{sca}}\left(\mathbf{r}_{m}, \theta_{l}\right)=\psi_{l}^{\mathrm{sca}}\left(\mathbf{r}_{m}\right) \sim \frac{(1+i) e^{i k\left|\mathbf{r}_{m}\right|}}{4 \sqrt{\pi k\left|\mathbf{r}_{m}\right|}} s\left(\theta_{m}, \theta_{l}\right)
$$

The complex amplitude of this field is proportional to the socalled scattering amplitude $s\left(\theta_{m}, \theta_{l}\right)=s_{m l}$, which depends on both the illumination angle $\theta_{l}$ and the detection angle $\theta_{m}$. The direct surface scattering problem corresponds to the calculus of the complex-valued array $s: m l \rightarrow s_{m l}$ for a given profile $\eta: x \rightarrow \eta(x)$. The inverse surface profiling consists in determining at best the roughness $\eta: x \rightarrow \eta(x)$ from the knowledge of the scattering amplitudes $s: m l \rightarrow s_{m l}$.

For an arbitrary rough surface, there exists no rigorous analytical solution for the direct surface wave scattering problem. Approximate models [14], based on simplifying physical assumptions, have first been developed, starting with Rayleigh for sound waves on gratings in the late nineteenth century. A commonly used theory is the so-called physical optics, where single scattering is assumed. It is in fact a family of models that share a similar form for the scattering amplitude

$$
s\left(\theta_{m}, \theta_{l}\right)=N\left(\theta_{m}, \theta_{l}\right) \int e^{-(x / g)^{2}} e^{-i Q_{z} \eta(x)} e^{-i Q_{x} x} \mathrm{~d} x,
$$

with $Q_{x}=k\left(\sin \theta_{m}-\sin \theta_{l}\right)$ and $Q_{z}=k\left(\cos \theta_{m}+\cos \theta_{l}\right)$. The geometrical coefficient $N\left(\theta_{m}, \theta_{l}\right)$ varies with the nature of the wave and of the scattering medium. Its expression depends on the physical assumptions on which the derivation is based: small height-to-wavelength ratio, small slope, tangent plane approximation, and so on. The key point with Eq. (2) is that $N\left(\theta_{m}, \theta_{l}\right)$ is independent of the roughness $\eta$. If a supplementary paraxial approximation $Q_{z}=2 k=4 \pi / \lambda$ is assumed, the integral in Eq. (2) turns to the FT of function $f(x)=e^{-(x / g)^{2}-2 i k \eta(x)}$. The so-called Fraunhofer estimate of the roughness is thus obtained by building $\tilde{f}\left(Q_{x}\right)=\frac{s\left(\theta_{m}, \theta_{l}\right)}{N\left(\theta_{m}, \theta_{l}\right)}$ and then by computing the original $f(x)$ by inverse FT. Then the height function $\eta(x)=\frac{\lambda}{4 \pi} \arg f(x)$ is retrieved. This simple inversion technique is used in most holographic or phase microscopy experiments. In this case, the transverse resolution is easily estimated from the spatial frequency span of $\tilde{f}$, which is accessible with the given illumination and detection angles. The resolution is tied to the single scattering assumption.

Most frequently, natural surfaces at optical wavelength are outside the validity domain of the physical optics. Multiple scattering does occur, and the relation between the profile $\eta$ : $x \rightarrow \eta(x)$ and the scattering amplitude $s: m l \rightarrow s_{m l}$ becomes much more complicated and cannot be directly inverted. This link can be mathematically represented by a nonlinear operator $\mathbf{F}$, the direct or forward scattering operator:

$$
\mathbf{F}: \eta \rightarrow s=\mathbf{F}_{\eta}
$$

This operator has no explicit expression, even if the direct surface wave scattering problem can nowadays be solved without any physical assumption by numerical methods $[\underline{11}, 15,16]$ such as the boundary integral equation method (BIE) [13] or the finite element method. The principle of the BIE is a good illustration of the implicitness of operator F. Following the BIE, the total field $\psi_{l}$ and its normal derivative $\partial_{n} \psi_{l}$ on the profile are the solutions of a boundary integral equation whose right-hand side is the incident field $\psi_{l}^{\text {inc }}$ on the profile. Once this equation is solved for a given incident angle $\theta_{l}$, the scattering amplitude can be computed via a scattering formula for any scattering angle $\theta_{m}$. The BIE is now a wellestablished method, and its numerical implementation for one-dimensional surfaces is for instance documented in [13].

\section{BIE}

We now detail these operators in the BIE context. With $\psi^{\text {inc }}\left(\mathbf{r}, \theta_{l}\right)=\psi_{l}^{\text {inc }}(\mathbf{r})$, the incident field for angle $\theta_{l}$, the total field writes $\psi_{l}(\mathbf{r})=\psi_{l}^{\text {inc }}(\mathbf{r})+\psi_{l}^{\text {sca }}(\mathbf{r})$. The scattering amplitude $s_{m l}$ is related to the value of the total field on the profile and its normal derivative $\partial_{n} \psi=\hat{\mathbf{n}} \cdot \operatorname{grad} \psi$ through scattering formula

$$
s_{m l}=-\int_{\Gamma}\left[\partial_{n} \psi_{l}(\mathbf{r})+i \mathbf{n} \cdot \mathbf{k}_{m} \psi_{l}(\mathbf{r})\right] \exp \left(-i \mathbf{k}_{m} \cdot \mathbf{r}\right) \mathrm{d} c
$$

where $\hat{\mathbf{n}}$ denotes the unit vector normal to the profile and directed toward air. The total field on $\Gamma$ and its normal derivative are the two surface unknowns of an integral equation: for any points $\mathbf{r}_{1}$ and $\mathbf{r}_{2}$ of $\Gamma$,

$$
\begin{aligned}
& \frac{1}{2} \psi_{l}\left(\mathbf{r}_{1}\right)-\int_{\Gamma} \partial_{n} G\left(\mathbf{r}_{1}, \mathbf{r}_{2}\right) \psi_{l}\left(\mathbf{r}_{2}\right) \mathrm{d} c_{2}+\int_{\Gamma} G\left(\mathbf{r}_{1}, \mathbf{r}_{2}\right) \partial_{n} \psi_{l}\left(\mathbf{r}_{2}\right) \mathrm{d} c_{2} \\
& =\psi_{l}^{\text {inc }}\left(\mathbf{r}_{1}\right),
\end{aligned}
$$

with $G\left(\mathbf{r}_{1}, \mathbf{r}_{2}\right)=+i H_{0}^{+}\left(k\left|\mathbf{r}_{2}-\mathbf{r}_{1}\right|\right) / 4$ the two-dimensional free space Green function and $H_{0}^{+}$the first kind Hankel function of zero order. The second equation required for solving the problem is provided by the boundary condition on $\Gamma$ and depends on the nature of the scattering medium and, for twodimensional problems, on the polarization case. The surface is assumed to be perfectly conducting, first for simplicity and second because, for such surfaces, the difference between the polarization cases is the most marked. Here the Dirichlet boundary condition corresponds to TE case, while the Neumann boundary condition is related to TM case:

$$
z=\eta(x) \Rightarrow \begin{cases}\psi_{l}(\mathbf{r})=0 & \mathrm{TE} \\ \partial_{n} \psi_{l}(\mathbf{r})=0 & \mathrm{TM}\end{cases}
$$

A single surface unknown remains: the surface current. The discretization method and the numerical resolution of Eq. (5) with condition Eq. (6) are detailed in [13]. The BIE is the numerical solution of a rigorous electromagnetic wave scattering theory. It includes all multiple scattering with no simplifying assumptions. The BIE constitutes a rigorous direct model. Thus, solving Eq. (5) with Eq. (6) for a given profile $\eta$ and applying Eq. (4) is equivalent to applying $\mathbf{F}$ on $\eta$. 


\section{INVERSE SURFACE WAVE SCATTERING PROBLEM}

In the general case, the link $\mathbf{F}: \eta \rightarrow s=\mathbf{F} \eta$ between the profile $\eta: x \rightarrow \eta(x)$ and the scattered field $s: m l \rightarrow s_{m l}$ is nonlinear. The inversion problem is then generally recasted as an optimization problem. In this section we describe the NewtonKantorovitch (NK) method [17] used to solve the nonlinear equation relating the data set of scattering amplitudes to the surface $\Gamma$. The NK method builds up iteratively the solution of Eq. (3) by successively solving the forward problem and a local linear inverse problem.

At each iteration step $n$, an estimate of the surface profile function is given by

$$
\eta^{n}=\eta^{n-1}+\delta \eta^{n}
$$

where $\delta \eta^{n}$ is an update correction that is obtained by solving in the least-squares sense the linearized forward problem

$$
\mathbf{D}^{n-1} \delta \eta^{n}=s-s^{n-1}
$$

with $s^{n-1}$ the scattered far field associated to the best available estimation of the surface $\eta^{n-1}$, while $\mathbf{D}^{n-1}$ is the Fréchet derivative of $\mathbf{F}$ at $\eta^{n-1}$.

For this local inverse problem, we make use of the Fréchet derivative $\mathbf{D}$ of operator $\mathbf{F}$ at a given height $\eta$. It links a variation $\delta \eta: x \rightarrow \delta \eta(x)$ of the profile $\eta$ to the first-order variation $\delta s: m l \rightarrow \delta s_{m l}$ of the scattering amplitude:

$$
\mathbf{D}: \delta \eta \rightarrow \delta s=\mathbf{D} \delta \eta=\lim _{t \rightarrow 0} \frac{\mathbf{F}(\eta+t \delta \eta)-\mathbf{F} \eta}{t} .
$$

The important point is that $\mathbf{D}$ is a linear operator. Its detailed derivation for electromagnetic waves and from the reciprocity theorem can be found in [18]. In the present case, the Fréchet derivative $\mathbf{D}$ of $\mathbf{F}$ writes as a curvilinear integral, whose expression depends on the polarization. It appears that $\delta s_{m l}=\delta s\left(\theta_{m}, \theta_{l}\right)$, the scattered field in direction $\theta_{m}$ for an incidence angle $\theta_{l}$, can be estimated from the surface currents $\psi_{l}$ or $\partial_{n} \psi_{l}$ related to incident $\theta_{l}$ but also involves the surface currents $\psi_{-m}$ or $\partial_{n} \psi_{-m}$ of the adjoint forward problem where the same surface is illuminated under incident angle $-\theta_{m}$. For the two polarization states, we have

$$
\begin{aligned}
& \mathrm{TE} \rightarrow \delta s_{m l}=-\int_{\Gamma} \partial_{n} \psi_{l}(\mathbf{r}) \partial_{n} \psi_{-m}(\mathbf{r}) \delta \eta(x) \mathrm{d} c \\
& \mathrm{TM} \rightarrow \delta s_{m l}=-\int_{\Gamma}\left\{k^{2} \psi_{l}(\mathbf{r}) \psi_{-m}(\mathbf{r})-\partial_{t} \psi_{l}(\mathbf{r}) \partial_{t} \psi_{-m}(\mathbf{r})\right\} \delta \eta(x) \mathrm{d} c .
\end{aligned}
$$

In the TM case, $\partial_{t} \psi=\hat{\mathbf{t}} \cdot \operatorname{grad} \psi$ denotes the tangential derivative of the total field on the profile.

Once the Fréchet derivative $\mathbf{D}$ is determined, the updated correction of the surface, which is the solution of Eq. (ㅁ), is obtained in the sense of the least mean squares. This solution minimizes the cost functional of the form

$$
\mathcal{F}(\delta \eta)=\|\mathbf{D} \delta \eta-\delta s\|^{2}
$$

Unfortunately, the problem of finding the solution of Eq. () is ill posed and needs regularization. This is achieved by minimizing a cost functional of the form

$$
\mathcal{F}(\delta \eta)=\|\mathbf{D} \delta \eta-\delta s\|^{2}+\mu^{2}\|\mathbf{R} \delta \eta\|^{2}
$$

where $\mu^{2}$ is the regularization parameter and $\mathbf{R}$ is the regularizing operator. Two operators have been studied for several examples, $\mathbf{R}=$ I which corresponds to the minimum norm solution of $\mathcal{F}$ described in Eq. (13) and $\mathbf{R}=\mathbf{S}$ the second order of the standard Tikhonov regularization [19]. Better results have been obtained by mixing both choices. Instead of minimizing the cost functional of Eq. (13), we use a mixture of zero- and second-order standard Tikhonov regularization and we minimize a cost functional of the form

$$
\mathcal{F}(\delta \eta)=\|\mathbf{D} \delta \eta-\delta s\|^{2}+\mu^{2}\left[(1-\alpha)\|\mathbf{I} \delta \eta\|^{2}+\alpha\|\mathbf{S} \delta \eta\|^{2}\right] .
$$

Equivalently, the minimum of $\mathcal{F}$ of Eq. (14) is the solution of the algebraic equation

$$
\left[\left(\mathbf{D}^{n-1}\right)^{\dagger} \mathbf{D}^{n-1}+\mu^{2}\left(\alpha \mathbf{S}^{\dagger} \mathbf{S}+(1-\alpha) \mathbf{I}\right)\right] \delta \eta^{n}=\left(\mathbf{D}^{n-1}\right)^{\dagger}\left(s-s^{n-1}\right),
$$

where $\mathbf{D}^{\dagger}$ stands for the adjoint of $\mathbf{D}$ and $\mu^{2}$ is the regularization parameter, which does not vary during the iterative process. $\mathbf{I}$ is the identity operator and $\mathbf{S}$ is the second-order derivative operator. The real $\alpha$ is the mixing parameter between the zero- and the second-order Tikhonov regularization, and its value is fixed to 0.95 for all numerical examples studied in this paper.

In practice, the regularization parameter $\mu^{2}$ is chosen by trial and error. For the numerical experiments reported here, $\mu^{2}$ takes the value that gives the lowest value of the cost function at the end of the iterations. This also corresponds qualitatively to the best reconstructions of the profiles.

\section{NUMERICAL RESULTS}

In the numerical study the wavelength is set to the optical wavelength of $\lambda=633 \mathrm{~nm}$. The surfaces are illuminated under an incidence angle varying from $-45^{\circ}$ to $+45^{\circ}$ with a $9^{\circ}$ step. The scattered field is collected at detection angles between $-45^{\circ}$ and $+45^{\circ}$ with a $1^{\circ}$ step. These conditions are representative of the experimental characteristics of ODTM setups used for microscopy applications [7,10]. With such angular ranges, the Rayleigh criterion for resolution is $\lambda /(4 \mathrm{NA})=$ $223 \mathrm{~nm}$ with $\mathrm{NA}=0.71$ the numerical aperture. The considered surface is $10 \mu \mathrm{m}$ long and consists in two identical bumps of width $80 \mathrm{~nm}$ and height $140 \mathrm{~nm}$ with a center-to-center of $200 \mathrm{~nm}$. The bumps are positioned at abscissas 500 and $700 \mathrm{~nm}$. The center of the illumination beam is at the origin of $x$ axis. Data for both polarizations are generated according to Eqs. $\underline{4}$ and $\underline{5}$ with boundary conditions Eq. $\underline{6}$, where the integral equations are cast into matrix-vector equations. In addition we used different mesh sizes for generating and inverting the data: the number of points on the profile is 4096 for the forward problem and 1024 for the inverse problem. The numbers of incident and diffracted angles are $L=$ 11 and $M=91$, respectively. The inversion process is stopped when convergence is reached, here after 600 iterations.

First, we apply the NK algorithm to the TE-polarized synthetic data from the two-bump profile, with the plane $\eta=0$ as initial guess and for several values of the Tikhonov parameter $\mu_{\mathrm{TE}}^{2}$. The reconstructed profiles are plotted in Fig. 1 for four values of the $\mu_{\mathrm{TE}}^{2}$ parameter. The bumps are correctly located 


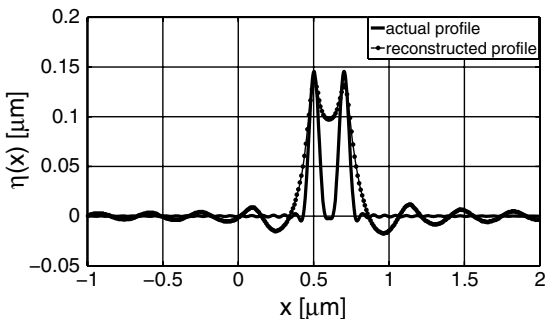

(a)

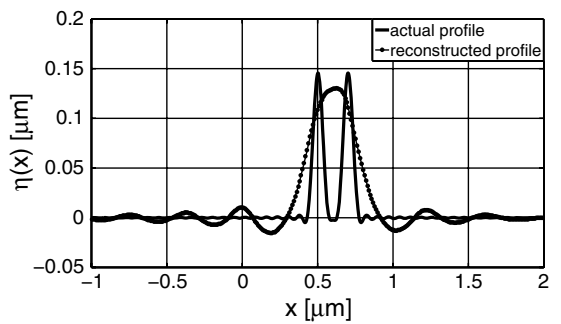

(c)

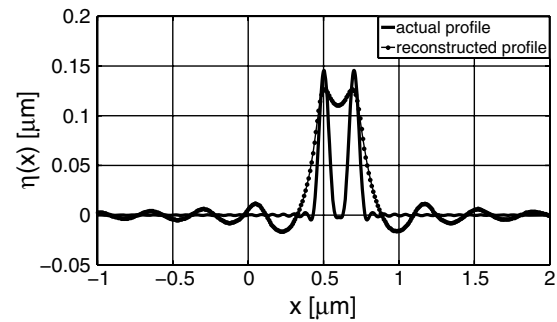

(b)

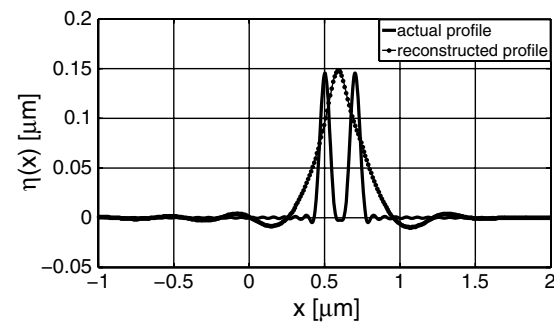

(d)

Fig. 1. Reconstructions of the profile at $\lambda=633 \mathrm{~nm}$, using the NK algorithm under TE polarization and starting from plane, for different values of the Tikhonov parameter: (a) $\mu_{\mathrm{TE}}^{2}=10^{7}$, (b) $\mu_{\mathrm{TE}}^{2}=10^{8}$, (c) $\mu_{\mathrm{TE}}^{2}=10^{9}$, and (d) $\mu_{\mathrm{TE}}^{2}=10^{11}$.

but are resolved only for the two lowest values $\mu_{\mathrm{TE}}^{2}=10^{7}$ and $\mu_{\mathrm{TE}}^{2}=10^{8}$. On plots $\underline{1(\mathrm{a})}$ and $\underline{1(\mathrm{~b})}$, the bumps are reconstructed accurately with correct values of height and width. The two bumps are not completely separated since the minimum value of the retrieved profile between the two bumps does not go under $100 \mathrm{~nm}$ for a bump height of $140 \mathrm{~nm}$. For values of the Tikhonov parameter smaller than $10^{7}$, the regularization is too weak to compensate the ill conditioning of the Fréchet derivative matrix $\mathbf{D}$, and the iteration process diverges or converges slowly toward unphysical solutions. For values larger than $10^{8}$ [Figs. $1(\mathrm{c})$ and $\underline{1(\mathrm{~d})}$ ], the problem is too strongly regularized and superresolution is lost, with performance comparable to that of methods based on single and paraxial scattering assumptions, such as the Fraunhofer approximation. Thus, the range of the regularization parameter $\mu_{\mathrm{TE}}^{2}$ for which superresolution is reached in TE polarization when the plane is used as initial guess is $\left[10^{7} ; 10^{8}\right]$.

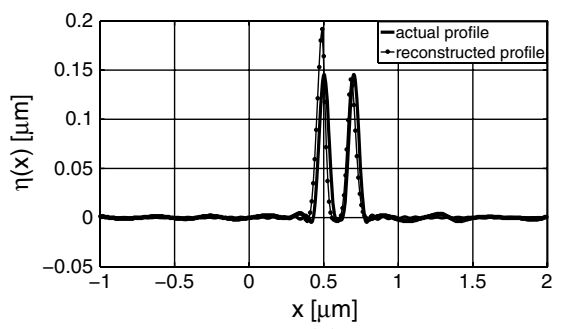

(a)

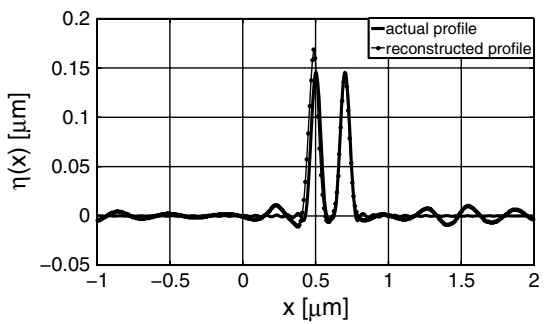

(c)
We now shift to the TM polarization case. It appears that synthetic data cannot be inverted in this polarization case when starting from the bare plane $\eta=0$. We also tried to use the Fraunhofer estimate as initial guess, but the Tikhonov parameter $\mu_{\text {TE }}^{2}$ for regularization has to be set precisely for the iterative process to converge. A considerable improvement is obtained when the surface profile retrieved in TE illumination is used as an initial guess for the inversion in the TM polarization case. Here, the TM-inverted surface depends on two regularization parameters: the value $\mu_{\mathrm{TE}}^{2}$ used to produce the initial guess from TE-polarized data and the value $\mu_{\mathrm{TM}}^{2}$ used to regularize the TM problem. All pairs $\left(\mu_{\mathrm{TE}}^{2}, \mu_{\mathrm{TM}}^{2}\right)$ for ranges $\mu_{\mathrm{TE}}^{2} \in\left[10^{5} ; 10^{12}\right]$ and $\mu_{\mathrm{TM}}^{2} \in\left[10^{7} ; 10^{14}\right]$ have been tested, and the best reconstruction is obtained for $\mu_{\mathrm{TE}}^{2}=10^{8}$ and $\mu_{\mathrm{TM}}^{2}=10^{9}$.

As one can see in Fig. 2(b), the retrieved bumps for TM polarization are separated, with the retrieved height between the

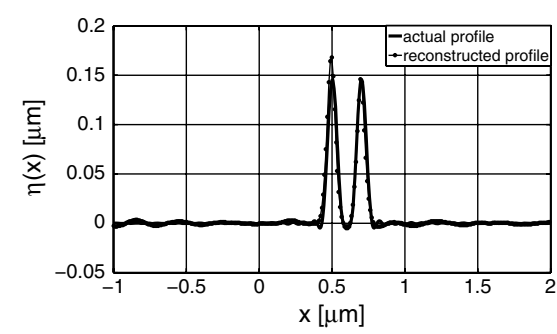

(b)

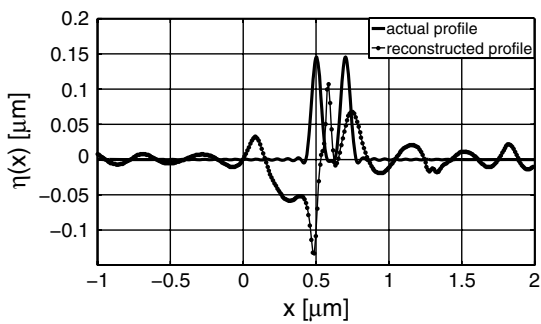

(d)

Fig. 2. Reconstructions of the profile at $\lambda=633 \mathrm{~nm}$ using NK algorithm under TM polarization with Tikhonov parameter fixed to $\mu_{\mathrm{TM}}^{2}=10^{9}$. The initial guess consists in the solution of the NK algorithm under TE polarization and starting from plane, for different values of the Tikhonov parameter: (a) $\mu_{\mathrm{TE}}^{2}=10^{7}$, (b) $\mu_{\mathrm{TE}}^{2}=10^{8}$, (c) $\mu_{\mathrm{TE}}^{2}=10^{9}$, and (d) $\mu_{\mathrm{TE}}^{2}=10^{11}$. 


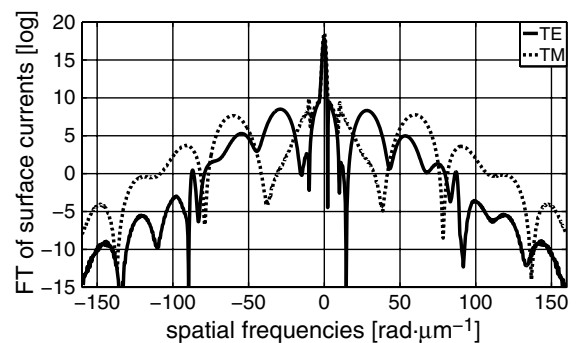

Fig. 3. Normalized spectral densities of the TE and TM surface currents.

two bumps being close to 0 . The rendering of the bases of the bumps is of much higher quality for that polarization case. The TE inversion is undoubtedly outperformed by the TM inversion in terms of resolution and general performance. We now discuss this result.

The presence of multiple scattering for bumps as high as $140 \mathrm{~nm}$, and its modelization in the iterative method, can explain why profiles are retrieved with superresolution [8,9]. However, the different behavior between TE and TM polarizations has to be commented. The comparison between the supports of the spectral densities of the surface currents, namely $\partial_{n} \psi$ in the TE case and $\psi$ in the TM case, show that (see Fig. 3), in TM polarization, higher frequencies can be retrieved. They have been normalized to share the same value at null frequency. However, without any other consideration, this would explain the obtained higher TM resolution only in the near field, since in the far field, spatial frequencies larger than the electromagnetic wavenumber are filtered. In order to better understand the role of multiple scattering in superresolution, we study the distance of interaction between two points of the surface. In integral Eq. 5, this distance of interaction $\left|\mathbf{r}_{1}-\mathbf{r}_{2}\right|$ appears explicitly in the Green's function. Thus, in the forward scattering problem, it is possible to suppress the interactions at a distance larger than a chosen value $d$. The associated scattered field is denoted $s_{m l}(d)$, while $s_{m l}$ denotes the rigorous scattered field, which includes all interactions on the surface. We express the so-called field difference parameter FD,

$$
\mathrm{FD}(d)=\sqrt{\frac{\sum_{m, l}\left|s_{m l}-s_{m l}(d)\right|^{2}}{\sum_{m, l}\left|s_{m l}\right|^{2}}},
$$

as a function of the interaction range $d$. This parameter is plotted in log scale as a function of $d$ for the two polarization cases [see Fig. 4(a)]. The results show a quite chaotic behavior from 0 to $300 \mathrm{~nm}$, when near interactions are the strongest. A

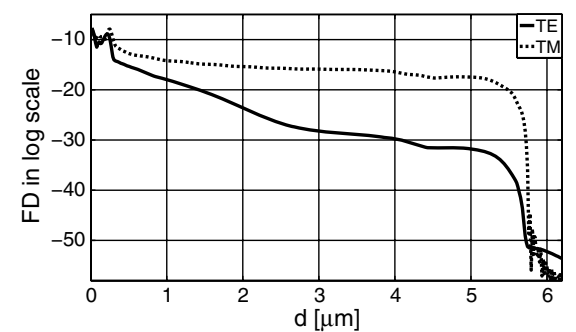

(a) monotonic region between 300 and $5.5 \mu \mathrm{m}$ is observed, but the TE curve decreases much faster than the TM curve. The values of FD finally vanish shortly before $d=6 \mu \mathrm{m}$, which roughly corresponds to the width of the beam footprint. This numerical experiment shows that the scattered field is much more sensitive to the far interactions between surface points in the TM case. These long-range interactions are responsible of the coupling of the high-frequency components of the surface currents into the low-frequency components that are collected by the detector. This effect appears to be stronger in TM polarization.

Another numerical experiment can be done by filtering the high spatial frequencies of the surface. If $k_{c}$ denotes the cut-off spatial frequency and $s_{m l}\left(k_{c}\right)$ the associated scattered field from the smoothed profile, the field difference can be expressed as a function of $k_{c}$ :

$$
\mathrm{FD}\left(k_{c}\right)=\sqrt{\frac{\sum_{m, l}\left|s_{m l}-s_{m l}\left(k_{c}\right)\right|^{2}}{\sum_{m, l}\left|s_{m l}\right|^{2}}} .
$$

This field difference $\operatorname{FD}\left(k_{c}\right)$ in the two polarization cases has been computed for cut-off frequencies up to $125 \mathrm{rad} \cdot \mu \mathrm{m}^{-1}$ in Fig. 4(b). The TM field appears to be more sensitive to the intermediate spatial frequencies of the profile over the range $\left[4 \mathrm{rad} \cdot \mu \mathrm{m}^{-1} ; 40 \mathrm{rad} \cdot \mu \mathrm{m}^{-1}\right.$. Stronger sensitivity to spatial frequencies higher than $40 \mathrm{rad} \cdot \mu \mathrm{m}^{-1}$ is not necessarily a good point for TE inversion: on one hand, these frequencies are far too high to be inverted, and on the other hand, they will be the first to be covered by noise in experimental data. Eventually, the behavior of the field difference at low cut-off frequency can explain the sensitivity of the NK algorithm to the initial guess when applied to surface scattering in the TM case. From zero to $k_{c}=4 \mathrm{rad} \cdot \mu \mathrm{m}^{-1}$, the TE curve is quickly decreasing, indicating its response to the lowest frequencies of the surface profile. On the same range, the TM curve is increasing. TM inversion is not adapted for retrieving the largest wavelengths of the profile that have to be supplied through the initial guess. Here we illustrate the sensitivity of the TM inversion to the initial guess by fixing the TM regularization parameter to the previously found optimal value $\mu_{\mathrm{TM}}^{2}=10^{9}$ and by varying the TE parameter $\mu_{\mathrm{TE}}^{2}$, which impacts the TM starting value of $\eta$. The four plots of Fig. 2 represent the profiles retrieved from TM data using the four TE profiles of Fig. 1 as initial estimates. Superresolved reconstructions are obtained for the three different values $10^{7}, 10^{8}$, and $10^{9}$ of the $\mu_{\mathrm{TE}}^{2}$ parameter. This indicates that this parameter does not have to be finely tuned. Moreover, in the case $\mu_{\mathrm{TE}}^{2}=10^{9}$, the bumps are not resolved in the TE case. We conclude that, when intended

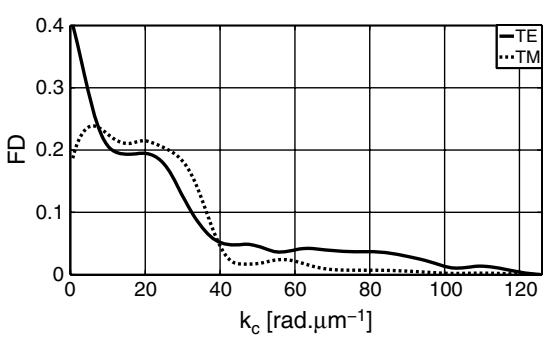

(b)

Fig. 4. Field difference over all incidence and scattering angles FD against (a) maximum interaction distance $d$, (b) cut-off spatial frequency of the filtered profile $k_{c}$. 


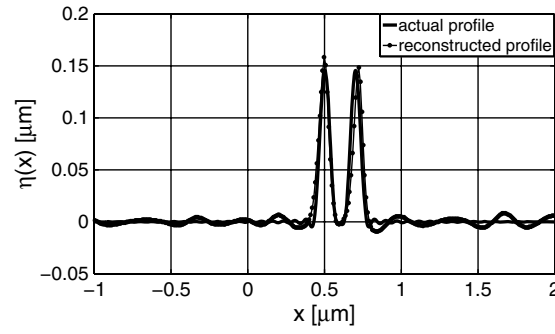

(a)

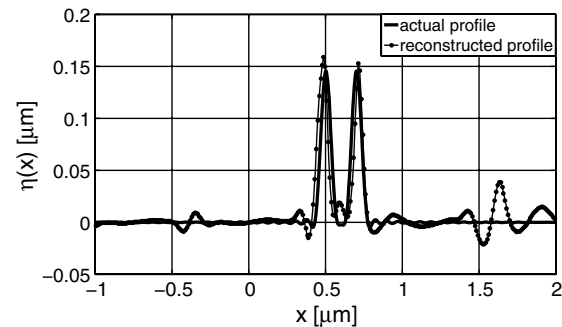

(c)

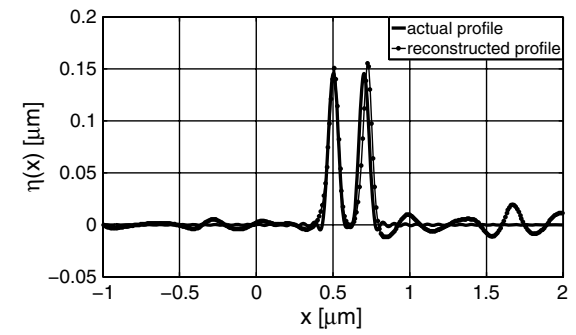

(b)

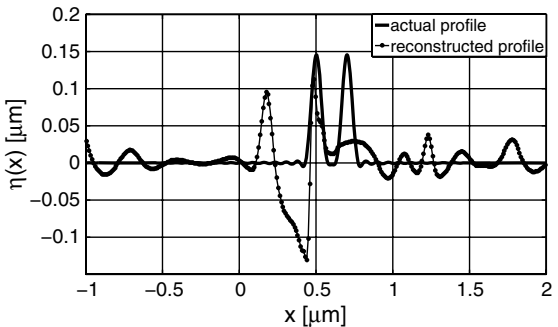

(d)

Fig. 5. Reconstructions at $\lambda=633 \mathrm{~nm}$ using NK algorithm under TM polarization with Tikhonov parameter fixed to $\mu_{\mathrm{TM}}^{2}=10^{9}$. Here the reconstructed surface obtained in the TE case with Tikhonov parameter fixed to $\mu_{\mathrm{TE}}^{2}=10^{9}$ is used as a starting solution. Various SNRs are considered. The SNRs are (a) 35, (b) 15, (c) 7, (d) 5 .

to be used as initial estimate for the TM inversion, the TE computation can be slightly overregularized. However, Fig. 2(d) shows that, when the initial guess is too strongly regularized, namely $\mu_{\mathrm{TE}}^{2}=10^{11}$, the method fails: here regularization suppresses a too large part of the profile spatial frequencies from the initial guess and prevents the TM inversion process to converge.

To check the robustness of the NK inversion method with respect to the presence of noise in the scattered field, a complex white noise is added to data. For each independent polarization and for a given signal-to-noise ratio (SNR), the noise is proportional to the amplitude of the real and imaginary parts of the scattered field. In order to maximize robustness to noise, we choose as parameter for the initial guess $\mu_{\mathrm{TE}}^{2}=$ $10^{9}=\mu_{\mathrm{TM}}^{2}$ that corresponds to the most regularized TE solution, which ensures the convergence of the TM inversion toward the actual profile when the data are free of noise. In Fig. 5, four values of the SNR are studied and applied to both TE and TM data. Inversion is very good for an SNR of 35, with no impact of the noise [Fig. 5(a)]. The inverse method shows a strong robustness to noise for an SNR of 15 and 7 [Figs. 5(b) and 5(c)]. The algorithm still converges for an SNR as low as 5 [Fig. 5(d)], but not toward the actual twobump profile: the retrieved profile shows important oscillations on its right side, and the right bump has vanished. If the left bump is correctly inverted, a fake hole and a supplementary bump appear on its left side. However, good results for an SNR of 7 or larger demonstrate the possible use of our inverse model on experimental data.

We have also tested the robustness of the reconstruction against errors of positioning angles of detection. For this purpose, we have performed numerical experiments with random errors on detectors' positions. Results have shown that reconstructions are not affected by a random angular error of $\pm 0.2^{\circ}$, which is an accuracy that can be easily reached with commercial rotation stages. This can be explained by the fact that the number of measured data is much higher than the number of unknowns in the inverse problem.

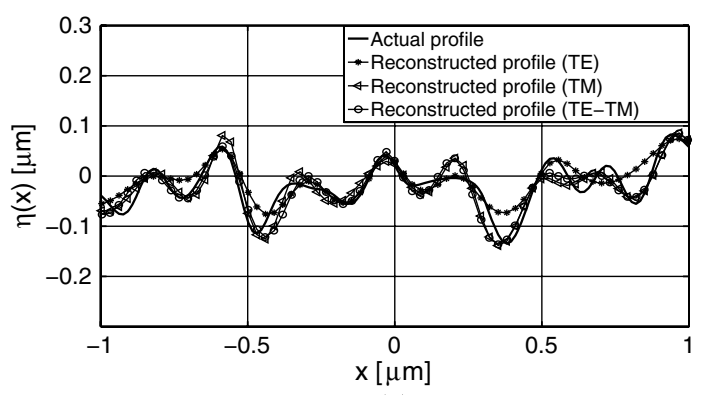

(a)

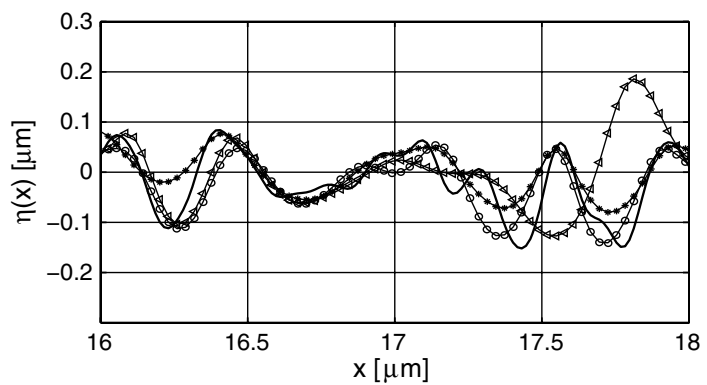

(b)

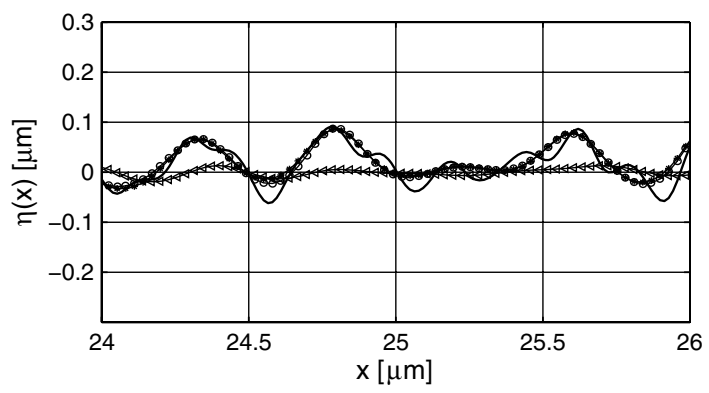

(c)

Fig. 6. Actual and reconstructed profiles using two different polarizations TE $\left(\mu_{\mathrm{TE}}^{2}=10^{9}\right)$ or TM, and two different initial guesses for the TM case. (a) Central part of the surface, (b) intermediate part, (c) edge part. The best reconstruction (circle line) is obtained in TM $\left(\mu_{\mathrm{TM}}^{2}=10^{14}\right)$ polarization when the initial guess is the reconstructed profile obtained in the TE $\left(\mu_{\mathrm{TE}}^{2}=10^{9}\right)$ case. 


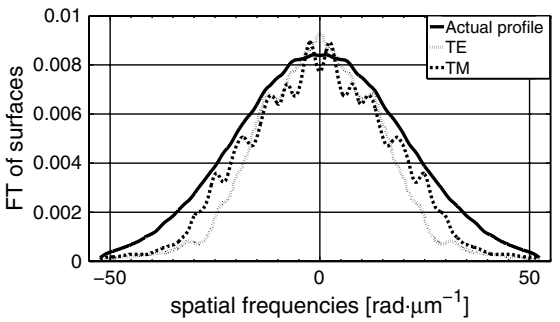

(a)

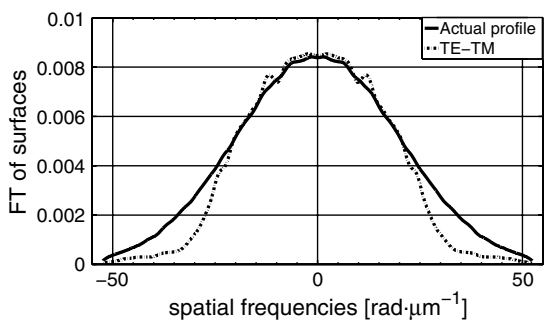

(b)

Fig. 7. FTs of an actual and the reconstructed profiles using two different polarizations TE $\left(\mu_{\mathrm{TE}}^{2}=10^{9}\right)$ or TM, and two different initial guesses for the TM case. (a) A bare plane $\eta=0$ as initial guess is used. (b) Reconstructed surface using the TM $\left(\mu_{\mathrm{TM}}^{2}=10^{14}\right)$ data and with as initial guess the final result of the reconstruction using $\mathrm{TE}\left(\mu_{\mathrm{TE}}^{2}=10^{9}\right)$ case.

In order to confirm the versatility of our approach, we present the inversion of a rough surface of long length. Its height root mean square is $60 \mathrm{~nm}$, and its correlation length is set to $\ell=100 \mathrm{~nm}$. A normal height distribution and a Gaussian height spectrum are given to the roughness. With a slope root mean square that corresponds to an angle of $40^{\circ}$, this surface is far too rough to be inverted under the Fraunhofer approximation [12]. The profile is $60 \mu \mathrm{m}$ long and sampled with 4096 points to compute the scattered field, which corresponds to data. Angles of incidence and scattering are the same as for the two-bump profile. For the inversion, the estimated profile is also $60 \mu \mathrm{m}$ long but counts only 2048 points. In Fig. (6), three reconstructions are reported and compared to the actual profile. The two first ones are obtained using solely TE and TM data, respectively, with the plane as initial guess, while for the third plot, the TE reconstruction is used as initial guess for TM inversion. The profile being too long to be shown clearly on a single plot, three views are presented. It appears that the TE reconstruction made with the plane as initial guess fails to retrieve the high spatial frequencies of the profile. The TM reconstruction made with the same initial guess fails to retrieve the low frequencies of the profile presents spurious peaks in the intermediate region of the profile and seems to be blind to its edges. When the reconstruction is made in TM polarization with the profile reconstructed in TE polarization as initial guess, the solution is much closer to the actual profile. The comparison is also brought to the Fourier space in Fig. 7. One can see that the low frequencies are better reconstructed in the TE case while the high frequencies are better retrieved in the TM case when the reconstructed surface obtained in TE polarization is used as starting solution.

These results show the importance of using both polarization cases for obtaining accurate and high resolution reconstructions.

\section{CONCLUSION}

In this paper we have presented an inversion method for reconstructing the profiles of rough perfectly conducting surfaces in the resonance domain from measures of the scattered field in TM polarization. We have shown that a strong improvement of the resolution can be obtained when the reconstructed surface obtained in the TE case is used as starting solution of the iterative reconstruction algorithm in TM polarization. We have also shown that a resolution beyond the Abbe-Rayleigh criterion can be reached when multiple scattering occurs within the surface. We have given a physical interpretation of the results by considering the distance of interaction between the scatterers on the surface. We have shown in particular that this distance is longer in the TM polarization than in the TE one, and we have studied its influence on the coupling between the spatial frequencies of the scattered field. Reconstructions of randomly rough surfaces have been presented. The robustness of the inversion method against noise and against angular errors of the detector's position have been also addressed.

The results presented in this paper are a step toward the development of a new optical profilometry technique that can deal with rough surfaces having strong slopes and strong roughness that cannot be characterized with the standard methods based on Linnik or Mirau interferometers.

\section{REFERENCES}

1. V. Lauer, "New approach to optical diffraction tomography yielding a vector equation of diffraction tomography and a novel tomographic microscope," J. Microsc. 205, 165-176 (2002).

2. O. Haeberlé, K. Belkebir, H. Giovaninni, and A. Sentenac, "Tomographic diffractive microscopy: basics, techniques and perspectives," J. Mod. Opt. 57, 686-699 (2010).

3. S. A. Alexandrov, T. R. Hillman, T. Gutzler, and D. D. Sampson, "Synthetic aperture Fourier holographic optical microscopy," Phys. Rev. Lett. 97, 168102 (2006).

4. M. Debailleul, B. Simon, V. Georges, O. Haeberlé, and V. Lauer, "Holographic microscopy and diffractive microtomography of transparent samples," Meas. Sci. Technol. 19, 074009 (2008).

5. M. Debailleul, V. Georges, B. Simon, R. Morin, and O. Haeberlé, "High-resolution three-dimensional tomographic diffractive microscopy of transparent inorganic and biological samples," Opt. Lett. 34, 79-81 (2009).

6. W. Choi, C. Fang-Yen, K. Badizadegan, S. Oh, N. Lue, R. R. Dasari, and M. S. Feld, "Tomographic phase microscopy," Nat. Methods 4, 717-719 (2007).

7. G. Maire, F. Drsek, J. Girard, H. Giovannini, A. Talneau, D. Konan, K. Belkebir, P. C. Chaumet, and A. Sentenac, "Experimental demonstration of quantitative imaging beyond Abbe's limit with optical diffraction tomography," Phys. Rev. Lett. 102, 213905 (2009).

8. K. Belkebir, P. C. Chaumet, and A. Sentenac, "Influence of multiple scattering on three-dimensional imaging with optical diffraction tomography," J. Opt. Soc. Am. A 23, 586-595 (2006).

9. F. Simonetti, "Multiple scattering: the key to unravel the subwavelength world from the far-field pattern of a scattered wave," Phys. Rev. E 73, 036619 (2006).

10. J. Girard, G. Maire, H. Giovannini, A. Talneau, K. Belkebir, P. C. Chaumet, and A. Sentenac, "Nanometric resolution using farfield optical tomographic microscopy in the multiple scattering regime,” Phys. Rev. A 82, 061801 (2010).

11. H. Giovannini, M. Saillard, and A. Sentenac, "Numerical study of scattering from rough inhomogeneous films," J. Opt. Soc. Am. A 15, 1182-1191 (1998). 
12. S. Arhab, G. Soriano, K. Belkebir, A. Sentenac, and H. Giovannini, "Full wave optical profilometry," J. Opt. Soc. Am. A 28, 576-580 (2011).

13. L. Tsang, J. A. Kong, K. H. Ding, and C. O. Ao, Scattering of Electromagnetic Waves: Numerical Simulations, Wiley Series in Remote Sensing (Wiley-Interscience, 2001).

14. T. M. Elfouhaily and C. A. Guérin, "A critical survey of approximate scattering wave theories from random rough surfaces," Waves Random Media 14, R1-R40 (2004).

15. M. Saillard and A. Sentenac, "Rigorous solutions for electromagnetic scattering from rough surfaces," Waves Random Media 11, 103-137 (2001)
16. K. F. Warnick and W. C. Chew, "Numerical simulation methods for rough surface scattering," Waves Random Media 11, R1-R30 (2001).

17. A. Roger, "Newton-Kantorovitch algorithm applied to an electromagnetic inverse problem," IEEE Trans. Antennas Propag. 29, 232-238 (1981).

18. A. Roger, "Reciprocity theorem applied to the computation of functional derivatives of the scattering matrix," Electromagnetics 2, 69-83 (1982).

19. A. N. Tikhonov, V. I. A. Arsenin, and F. John, Solutions of Ill-Posed Problems (Winston, 1977). 